\title{
NUEVO ENFOQUE EN LA ENSEÑANZA DE LAS MATEMÁTICAS, EL MÉTODO ABN
}

\author{
Mª del Pilar Díaz-López \\ Nuria del Mar Torres López \\ Ma Carmen Lozano Segura \\ Universidad de Almería
}

https://doi.org/10.17060/ijodaep.2017.n1.v3.1012

Fecha de Recepción: 29 Marzo 2017

Fecha de Admisión: 1 Abril 2017

\section{RESUMEN}

Las matemáticas, como afirma Le Lionnais (1976, p.15). "constituyen una de las formas más reveladoras y sorprendentes del pensamiento humano". Por ello, "hacer matemáticas", en cualquier nivel, es (o debería ser) siempre una tarea humana apasionante, enriquecedora, única. Objetivo: realizar una búsqueda bibliográfica que aborde programas de intervención, experiencias en las aulas de infantil/primaria o ejemplos de buenas prácticas en las principales bases de datos de Ciencias de la Educación y Psicología. Metodología: se ha realizado una búsqueda bibliográfica en seis bases de datos: PubMed, Scopus, Dialnet, ERIC, ProQuest EDUCATION JOURNALS y Psicodoc de los últimos diez años. Los descriptores fueron: "ABN", "matemáticas", "educación infantil" y "educación primaria" en inglés y español, combinados por el conector AND. Se han incluido algunos artículos que contenían estudios con grupo control y experimental y algunas revisiones 0 trabajos fin de máter 0 grado. Resultados: Ios artículos y revisiones incluidas contenían un total de 444 participantes. Describen las características, tipo de intervención, actividades desarrolladas, seguimiento, resultados y conclusiones en los distintos estudios. Dichas investigaciones demuestran que la enseñanza de las matemáticas con el método ABN presenta beneficios en el alumnado, permite mejorar el cálculo y la resolución de problemas, entre otras habilidades. Conclusiones: la literatura científica y la publicación de estudios experimentales en relación al tema de estudio es aún escasa, no obstante se muestran las bondades de la metodología ABN frente a la metodología tradicional en diversas prácticas y algunos estudios.

Palabras claves: metodología ABN, educación infantil, educación primaria y matemáticas.

\section{INTRODUCCIÓN}

Las matemáticas, como afirma Le Lionnais (1976, p.15). "constituyen una de las formas más reveladoras y sorprendentes del pensamiento humano". Por ello, "hacer matemáticas", en cualquier nivel, es (o debería ser) siempre una tarea humana apasionante, enriquecedora, única. 
Las raíces de las matemáticas nos llevan a las antiguas y grandes civilizaciones de todos los continentes del planeta. En la Cultura Inca estaban relacionadas con la astronomía, y en la cultura egipcia ya realizaban complejos cálculos arquitectónicos en la construcción de las pirámides. Además las culturas clásicas y árabes tuvieron grandes matemáticos que desarrollaron complejos sistemas. Gracias a estos sistemas se ha desarrollado la ciencia empírica y tecnológica tal y como la conocemos hoy día

En educación infantil las matemáticas juegan un papel fundamental, a pesar de que en ocasiones se considera socialmente que los contenidos matemáticos de esta etapa educativa son simples, lo cierto es que son la base de adquisición de un complejo sistema que hasta ahora venían enseñándose de forma mecanizada y sin relación útil y directa con la vida diaria.

Por ejemplo, actividades como el conteo o la designación de números parecen actividades cotidianas y naturales para las que poco habría que saber, ya que la mayoría de nosotros las hacemos de forma automática, sin embargo estas acciones que nos parecen evidentes necesitan de una preparación. En efecto, no es nada trivial construir con sentido el número natural o llevar a cabo la actividad de contar o medir, así como en las relaciones entre ellas, por no decir las condiciones necesarias para que se puedan realizar con sentido. Los fundamentos de las matemáticas son en realidad altamente complejos, dejando de parecer trivial a partir del momento en el que uno se detiene a analizarlos, a problematizarlos y a reconstruirlos- condición necesaria para gestionar adecuadamente su proceso de aprendizaje.

La escuela infantil es, además, el lugar donde se inicia el desarrollo de la personalidad racional del alumno, por ello debe facilitar ámbitos donde se generen los comportamientos sociales relativos a la toma de decisiones y al establecimiento de la verdad. La búsqueda de la verdad a través de la prueba es el instrumento básico para la constitución de la racionalidad human. Esta formación fundamental se comienza a gestar muy precozmente, y de forma muy significativa, a través de la actividad matemática en la escuela infantil.

Brousseau (1997) señala que a través de las matemáticas el niño de infantil comienza a descubrir la gestión personal y social de la verdad.

Cuando un niño comienza a observar que existen hechos que se repiten sistemáticamente, de los que deducen conclusiones que pueden compartir con todos, que son ciertas siempre, que no son sustituidas por otras de modo arbitrario, en las que estamos siempre todos de acuerdo, que podemos y debemos validar y probar, que no son meras convicciones sociales, convenios más 0 menos estables, normas válidas según las ocasiones... Cuando un niño comienza a aproximarse a estas constataciones, está gestionando algo tan fundamental en la vida como es la verdad. Los conocimientos matemáticos surgen así, en este nivel educativo, como el fundamento necesario, sólido y universal de la verdad.

El aprendizaje matemático permite al niño/a organizar mentalmente sus impresiones de las cosas (números), sus atributos (cantidad, forma, características...) y relaciones (comparación, correspondencia, posición espacial). Para conocer la realidad, el niño/a realiza colecciones, seriaciones empíricas, cuenta sin conservar la cantidad, se orienta en el espacio a partir del propio cuerpo y construye las primeras representaciones topológicas. El aprendizaje de las matemáticas resulta complejo por la necesidad de abstracción, dificultad propia de la etapa.

En la enseñanza de las matemáticas tradicionalmente se han desarrollado mecanismos memorísticos y automáticos que enseñaban a realizar cálculos siguiendo fórmulas estancas y alejadas de la cotidianidad, en los últimos años se viene implantando en las aulas de infantil un método denominado ABN "Algoritmos Abiertos Basados en Números". El autor del Método ABN es Jaime Martínez Montero, maestro y doctor en Filosofía y Ciencias de la Educación. Ha escrito diversos 
libros que tratan la temática y señala que esta metodología se caracteriza principalmente por su carácter abierto, donde tienen cabida distintas formas de resolver un mismo problema u operación. La base del aprendizaje es el número y no la cifra. Para adquirir el concepto de número el alumnado se acercará y manipulará distintos materiales, contará, descompondrá, compondrá y realizará agrupaciones, repartos, emparejamientos de conjuntos de distintos elementos analizando semejanzas y diferencias de forma que interioricen el concepto pudiendo aplicarlo a la cadena numérica.

En uno de sus libros junto con Concepción Sánchez Cortes (2011), tratan las distintas técnicas significativas para que los niños adquieran conceptos matemáticos como el conteo, la suma, la resta, la multiplicación y la división.

\section{OBJETIVO}

Realizar una búsqueda bibliográfica que aborde programas de intervención, experiencias en las aulas de infantil o ejemplos de buenas prácticas en las principales bases de datos de Ciencias de la Educación y Psicología.

\section{METODOLOGÍA}

En primer lugar se realizó una búsqueda bibliográfica en seis bases de datos: PubMed, Scopus, Dialnet, ERIC, ProQuest EDUCATION JOURNALS y Psicodoc de los últimos diez años. Los descriptores fueron: "ABN", "matemáticas", "infantil" en inglés y español, combinados por el conector AND.

Se seleccionaron estudios cuasiexperimentales, estudios descriptivos, trabajos fin de grado y máster y revisiones sistémicas publicadas entre los años 2007 y 2017, en base a los siguientes criterios:

- Criterios de inclusión: estudios con programas de matemáticas dirigidos a alumnado de infantil y/o primaria, que abordasen de forma específica sus beneficios y la descripción de actividades realizadas.

- Criterios de exclusión: investigaciones que no definiesen las actividades llevadas a cabo en el programa, ni sus posibles efectos o beneficios; 0 que no fuesen dirigidos a esa población. Que los artículos tuviesen una antigüedad mayor a 10 años.

Un total de 110 artículos seleccionados por medio de esta estrategia, fueron analizados tanto por el título como por el resumen. En los casos, donde la investigación se consideró potencialmente relevante (10 artículos), se hizo un lectura crítica de la publicación a texto completo, basándose en el análisis exhaustivo de la intervención (tipo, modalidad, frecuencia, duración, muestra, objetivos, resultados, etc.).

\section{RESULTADOS}

Los artículos y revisiones incluidas contenían un total de 444 participantes donde se describen las características, tipo de intervención, actividades desarrolladas, seguimiento, resultados y conclusiones.

En esta búsqueda bibliográfica se halló un estudio realizado con 128 estudiantes de primer curso. El alumnado del grupo experimental (instruido con el método ABN) muestra mejores resultados en el cálculo con la memoria de trabajo, aplicando mentalmente las representaciones visoespaciales en las que han sido entrenados (Estíbaliz, Aragón-Mendizábal, Canto-López, MarchenaConsejero, Navarro-Guzmán y Aguilar-Villagrán, 2017)

Otro estudio cuasi-experimental llevado a cabo en dos centros escolares de Córdoba, pone de manifiesto la mejora en la competencia matemática del grupo experimental (grupo que siguió la metodología basada en los algoritmos ABN), esta metodología mejora los aspectos formales del aprendizaje matemático pero también los no formales. 


\section{NUEVO ENFOQUE EN LA ENSEÑANZA DE LAS MATEMÁTICAS, EL MÉTODO ABN}

Por otro lado, se describe un estudio que consistió en el análisis de los logros alcanzados por 210 alumnos de cuatro colegios públicos de la Bahía de Cádiz. En el artículo se centran en los resultados obtenidos por dos grupos de segundo de primaria. Se concluye nuevamente con el éxito en el desarrollo de la competencia matemática que aporta el algoritmo ABN (Martínez, 2011).

Finalmente se han analizado varios trabajos fin de grado y fin de máster en los que se hace un análisis más descriptivo del método, las condiciones para su implantación e implementación, las actividades que se pueden realizar etc.

\section{DISCUSIÓN Y CONCLUSIONES}

En los últimos años, la proliferación de estudios basados en las prácticas innovadoras y en concreto en la enseñanza de las matemáticas mediante el Algoritmo Abierto Basado en Números ha aumentado. En esta revisión de la literatura, la mayoría de los artículos que se han analizado se gestaron en los últimos tres años

Se pone de manifiesto el creciente interés actual por la obtención de información sobre los beneficios de la enseñanza de metodologías innovadoras con métodos como el ABN para disminuir las dificultades de aprendizaje.

La mayoría de las investigaciones revisadas sugieren que es evidente que la intervención con programas adaptados al pensamiento y desarrollo del niño van a generar beneficios en su aprendizaje. No obstante es necesario seguir investigando en este campo ya que la literatura científica referente a esta temática es escasa.

\section{REFERENCIAS BIBLIOGRÁFICAS}

Aragón-Mendizábal, E., Canto-López, M. C., Marchena-Consejero, E., Navarro-Guzmán, J. I., y Aguilar-Villagrán, M. (2017). Perfil cognitivo asociado al aprendizaje matemático con el método algoritmo abierto basado en números. Revista de Psicodidáctica, 22(1), 54-59.

Bracho-López, R., Gallego-Espejo, M. del C., Adamuz-Povedano, N., Jiménez-Fanjul, N. (2014). Impacto Escolar de la Metodología Basada en Algoritmos ABN en Niños y Niñas de Primer Ciclo de Educación Primaria. Revista Iberoamerican de Educación Matemática, 39, 97-109.

Brousseau, G. (1997). Theory of Didactical Situations in Mathematics. Dordrecht: Kluwer.

LE LIONNAIS, François. 1976. "La belleza en matemáticas" en LE LIONNAIS, F. y colaboradores. Las grandes corrientes del pensamiento matemático. pp. 464-494. Ed. Universitaria de Buenos Aires. Buenos Aires (Argentina), $3^{\underline{a}}$ ed

Martínez, J. (2011). El método de cálculo abierto basado en números (ABN) como alternativa de futuro respecto a los métodos tradicionales cerrados basados en cifras (CBC). Bordón, 63(4), 95-110.

Martínez, J. y Sánchez C. (2011). Desarrollo y mejora de la inteligencia matemática en educación infantil. Wolters Kluwer Educacion. 